\title{
NK cells and Lipoxin A4 promote resolution of eosinophilic inflammation after nasal allergen challenge
}

\author{
Anh Poirot ${ }^{1}$, Guillaume Wacht ${ }^{2}$, Lehalle Christine ${ }^{3}$, Philippe Saas ${ }^{4}$, Nelly Frossard ${ }^{5}$, \\ Bernard Geny ${ }^{6}$, Frederic de Blay ${ }^{7}$, and Cindy Barnig ${ }^{8}$ \\ ${ }^{1}$ Hopitaux universitaires de Strasbourg \\ ${ }^{2}$ EA 3072, University of Strasbourg \\ ${ }^{3}$ UMR 7200 CNRS / Université de Strasbourg, Laboratoire d'Innovation Thérapeutique \\ and LabEx MEDALIS, Faculté de Pharmacie \\ ${ }^{4}$ Univ. Bourgogne Franche-Comté, INSERM, EFS BFC, UMR1098, Interactions \\ Hôte-Greffon-Tumeur/Ingénierie Cellulaire et Génique, LabEx LipSTIC \\ ${ }^{5}$ CNRS-Université de Strasbourg and LabEx Medalis, Faculté de Pharmacie \\ ${ }^{6}$ Hôpitaux Universitaires de Strasbourg \\ ${ }^{7}$ Department of Chest Disease, University Hospital of Strasbourg \\ ${ }^{8}$ University Hospital Centre Besancon
}

May 18, 2021

\begin{abstract}
Background: Resolution of inflammation is now recognized as a tightly regulated and active process. Lipoxins (LX) are lead members of a larger family of specialized pro-resolving mediators with unique anti-inflammatory and pro-resolving properties. Recent studies implicated natural killer (NK) cells in the resolution of allergic airway inflammation, notably in promoting eosinophil apoptosis. The aim of the study was to better understand the pro-resolving actions of NK cells and LXA4 during allergic eosinophilic airway inflammation. Methods: 20 subjects with grass pollen allergic rhinitis were included. A nasal provocation test with either a single grass pollen allergen threshold dose or diluent was used. Nasal lavage fluid and cells were collected at baseline and at different time points after challenge. For in vitro assays, eosinophils were incubated with NK cells. Results: We observed that NK cells were recruited to the nasal mucosa shortly after the initiation of the allergic inflammatory response. This recruitment correlated with eosinophilic inflammation. In vitro assays demonstrated that direct contact and a combined action of CD56bright and CD56dim NK cells were needed to promote autologous eosinophil apoptosis. We furthermore observed that local LXA4 production correlated with the peak of neutrophil nasal mucosal infiltration, suggesting a potential role of neutrophils in LXA4 biosynthesis during the early phase of the allergic inflammatory response. Last, LXA4 appeared as essential to inhibit the in vitro release of eosinophil superoxide triggered by NK cells. Conclusion: Together, these findings indicate a synergistic role for NK cells and LXA4 in the resolution of allergic eosinophilic inflammation.
\end{abstract}

\section{NK cells and Lipoxin $\mathbf{A}_{4}$ promote resolution of eosinophilic inflammation}

\section{after nasal allergen challenge}

Short title: Resolution of eosinophilic inflammation

Anh Poirot, $\mathrm{BSc}^{1}$; Guillaume Wacht, $\mathrm{MD}, \mathrm{MSc}^{2}$; Christine Lehalle, $\mathrm{MSc}^{3}$; Philippe Saas, $\mathrm{PhD}^{4}$; Nelly Frossard, $\mathrm{PhD}^{3}$; Bernard Geny, MD, $\mathrm{PhD}^{2}$; Fréderic de Blay, $\mathrm{MD}^{1}$; Cindy Barnig, $\mathrm{MD}, \mathrm{PhD}^{4,5}$

1 Department of Chest Disease, University Hospital of Strasbourg, Strasbourg, France 
${ }^{2}$ EA 3072, University of Strasbourg, France

${ }^{3}$ UMR 7200 CNRS / Université de Strasbourg, Laboratoire d'Innovation Thérapeutique and LabEx MEDALIS, Faculté de Pharmacie, Strasbourg, France

4 Univ. Bourgogne Franche-Comté, INSERM, EFS BFC, UMR1098, Interactions Hôte-GreffonTumeur/Ingénierie Cellulaire et Génique, LabEx LipSTIC, F-25000 Besançon, France

${ }^{5}$ Department of Chest Disease, University Hospital of Besançon, Besançon, France

Address correspondence to :

Cindy Barnig

Univ. Bourgogne Franche-Comté,

INSERM, EFS BFC, UMR1098,

Interactions Hôte-Greffon-Tumeur/Ingénierie Cellulaire et Génique,

LabEx LipSTIC,

F-25000 Besançon, France

Tel: +33 381668802

e-mail :

cindy.barnig@univ-fcomte.fr

\section{Abstract}

Background : Resolution of inflammation is now recognized as a tightly regulated and active process. Lipoxins (LX) are lead members of a larger family of specialized pro-resolving mediators with unique antiinflammatory and pro-resolving properties. Recent studies implicated natural killer (NK) cells in the resolution of allergic airway inflammation, notably in promoting eosinophil apoptosis. The aim of the study was to better understand the pro-resolving actions of NK cells and LXA 4 during allergic eosinophilic airway inflammation.

Methods: 20 subjects with grass pollen allergic rhinitis were included. A nasal provocation test with either a single grass pollen allergen threshold dose or diluent was used. Nasal lavage fluid and cells were collected at baseline and at different time points after challenge. For in vitro assays, eosinophils were incubated with NK cells.

Results : We observed that NK cells were recruited to the nasal mucosa shortly after the initiation of the allergic inflammatory response. This recruitment correlated with eosinophilic inflammation.In vitro assays demonstrated that direct contact and a combined action of CD $56^{\text {bright }}$ and CD56 $6^{\text {dim }}$ NK cells were needed to promote autologous eosinophil apoptosis. We furthermore observed that local $\mathrm{LXA}_{4}$ production correlated with the peak of neutrophil nasal mucosal infiltration, suggesting a potential role of neutrophils in $\mathrm{LXA}_{4}$ biosynthesis during the early phase of the allergic inflammatory response. Last, $\mathrm{LXA}_{4}$ appeared as essential to inhibit the in vitro release of eosinophil superoxide triggered by NK cells.

Conclusion : Together, these findings indicate a synergistic role for NK cells and $\mathrm{LXA}_{4}$ in the resolution of allergic eosinophilic inflammation.

Key Words : eosinophil, lipoxin, nasal allergen challenge, Natural Killer cells

Word count : 3487 words

Introduction 
Allergic airway diseases, such as rhinitis and asthma, are one of the most common chronic inflammatory respiratory diseases in the world (1). The pathophysiology of allergic airway diseases involves a typical immunoglobulin (Ig) E-mediated type 2 immune response triggered in sensitized subjects following inhalation of aeroallergens with subsequent eosinophilic infiltration of the respiratory mucosa (2). Although the mechanisms underlying the pathology and treatment of allergic airway inflammation have been widely studied, many aspects remain unclear, notably how allergic inflammation effectively resolves in the airways.

The resolution of inflammation is now recognized as a tightly regulated and active process (3). From the apoptosis and clearance by myeloid cells of activated inflammatory cells to the release of pro-resolving molecules, resolution enables inflamed tissues to return to homeostasis. Non-resolving inflammation is suspected as a key factor in the pathogenesis of numerous chronic inflammatory diseases, including allergic rhinitis and asthma (4).

Lipoxins (LX) are lead members of a larger family of specialized pro-resolving mediators with unique antiinflammatory and pro-resolving properties (5). LX derive from essential fatty acids and are generatedvia biosynthetic routes engaged during cell-cell interactions (for example when infiltrating neutrophils interact with tissue-resident cells in inflamed target organs) (5). Previous animal studies showed that LX can decrease allergic inflammation $(6,7)$. Moreover, low lipoxin production has been observed in patients with severe and uncontrolled asthma $(8,9)$.

Natural Killer (NK) cells are well-known players in the control and elimination of virally-infected or cancer cells (10). But several animal studies also implicated NK cells in the resolution of allergic inflammation, notably in dampening eosinophilic inflammation (11). Previous data demonstrated that NK cells are able to induce the apoptosis of human autologous eosinophils in vitro $(12,13)$. In contrast, a potential activator effect of NK cells on eosinophils has also been reported, notably by increasing reactive oxygen species (ROS) expression by eosinophils in co-cultures (13).

The aim of the study was to better understand the pro-resolving actions of NK cells and $\mathrm{LXA}_{4}$ during a self-limited allergic respiratory inflammatory response by using a standardized nasal allergen provocation test with a single threshold dose. We were able to identify a synergistic role for NK cells and $\mathrm{LXA}_{4}$ in mediating resolution of allergic eosinophilic inflammation.

\section{Methods}

\section{Study participants}

The study population consisted of 20 subjects (mean [SD] age, years (range); 28.0 [7.1] (19-46); ten men and ten women) suffering from seasonal allergic rhinitis to grass pollen. The study was approved by the local Research Ethics Committee. Informed written consent was obtained from all subjects before their participation.

Inclusion criteria were a history of summer grass pollen allergic rhinitis for at least 2 years confirmed by a positive skin prick test to grass pollen extract (Stallergenes) i.e. $>3 \mathrm{~mm}$ weal compared with the negative control and positive specific IgE-antibodies to grass pollen, assessed by Phadia (mean [SD], kU/L (range); $16.5 \pm 23.4$ (0.4-100). Patients were excluded if they had current symptoms of rhinitis, other nasal or systemic disease, asthma requiring regular inhaled corticosteroids, abnormal pulmonary function, other respiratory conditions, current medication including anti-allergic medication, specific immunotherapy in the previous 5 years, smoking history in the past 6 months, were pregnant or breastfeeding women. None of the subjects had exposure to other relevant respiratory allergens and to avoid natural allergen provocation, all subjects were studied outside the grass-pollen season. At screening, participants were examined, including a nasal examination to rule out any nasal disease or evidence of active rhinitis. Throughout the whole study period, subjects were not allowed to take any medication.

\section{Nasal allergen challenge procedure}

A first nasal allergen challenge was performed to titrate the allergen dose eliciting a positive response. 
Briefly, nasal allergen challenge was performed as previously described by administering increasing doses of a grass pollen allergen extract (Stallergenes Greer) every 10 minutes by means of a nasal pump spray delivery device (14). A Lebel score $>5$ was considered as a positive response (15). After a washout period of 4 weeks, patients underwent two nasal allergen challenges with either a single threshold dose of the grass pollen allergen extract (Stallergenes Greer) or a diluent (saline solution) in a randomized order at least 4 weeks apart. All the challenges were performed outside the grass pollen season, and none of the subjects was exposed to any other relevant respiratory allergens.

\section{Total nasal symptom score}

Total nasal symptom scores (TNSS) were recorded at baseline just before the challenge, 15 and 30 min after challenge, and then again 1, 6, 24, 48 and $72 \mathrm{~h}$ after challenge (14). TNSS comprised the sum of the score of 4 nasal symptoms (rhinorrhea, nasal obstruction, sneezing, and nasal itching) scored from 0 to 3 ( $0=$ none, $1=$ mild, $2=$ moderate and $3=$ severe).

\section{Nasal lavage procedure and processing}

Nasal lavage samples were collected at baseline, and 1,6,24, 48 and $72 \mathrm{~h}$ post-challenge as described by Naclerio et al . (16). Briefly, $5 \mathrm{ml}$ of a prewarmed $\left(37^{\circ} \mathrm{C}\right)$ saline solution $(0.9 \% \mathrm{w} / \mathrm{v} \mathrm{NaCl})$ was instilled into each nostril with a $10 \mathrm{ml}$ syringe, attached by tubing to a nasal adapter. The subjects were seated in a forward-flexed neck position to prevent fluid from reaching the nasopharynx. The fluid was withdrawn into the syringe and flushed back into the nasal cavity 3 times. Mean [SD] recovery of nasal lavage fluid (10 ml for each washing) was $6.9 \mathrm{ml}[0.9]$ for allergen and $6.8 \mathrm{ml}$ [0.9] for placebo. The nasal lavage samples were kept on ice and then immediately centrifuged at $4^{\circ} \mathrm{C}$ for $10 \mathrm{~min}$ at $300 \mathrm{xg}$ to generate a supernatant which was stocked at $-80^{\circ} \mathrm{C}$ for later analysis. The cell pellet was resuspended in Dulbecco's phosphate-buffered saline (DPPS) (Lonza). Total numbers of leukocytes were counted by using an improved Neubauer haemocytometer and cell numbers were normalized to nasal lavage volume.

\section{Total nasal lavage protein levels}

Total protein content in nasal lavage supernatants was measured in duplicate in 96-well plates by a standard bicinchoninic acid assay (BCA) assay according to the manufacturer's instructions (Sigma-Aldrich).

\section{Flow Cytometry analysis}

Freshly processed nasal lavage cells were divided into 0.2 to $1 \times 10^{6}$ cells per tube, resuspended in $50 \mu$ of phosphate-buffered saline (PBS) (Lonza) and incubated in the presence of antibodies for 20 minutes on ice in the dark. Cells were then washed with PBS and resuspended for analysis. The following antibodies to human cell membrane proteins were used: CD45-PE Cy7, CD14-APC eFluor, CD16-FITC, CD56-APC, CD3PerCPCy5.5 (all from eBioscience, San Diego, CA) and NKp46-PE (9E2) (BioLegend). Data was acquired on an ARIA flow cytometer (Becton Dickinson) with FACSDiva acquisition software (Becton Dickinson BioSciences, Mississauga, Ontario, Canada) and analyzed using FlowJo flow cytometric analysis software version 10.1 (Tree Star, Ashland, OR). Leukocyte populations were identified in nasal lavage samples as described previously for induced sputums (17). Briefly, doublets were excluded by gating on forward scatter (FSC)-H and FSC-A, followed by gating on CD45 positive cells to discriminate leukocytes from contaminating squamous-epithelial cells. Cellular debris were then gated out on the basis of FSC and side scatter (SSC). Specific populations were identified on the basis of marker expression as well as size and granularity. Respective gating strategy is outlined in Fig $2 \mathrm{~A}$. For nasal lavage samples, as many events as possible were collected. For compensation control, BD CompBeads (BD PharMingen) were used.

\section{Lipoxin $\mathbf{A}_{4}$ nasal lavage levels}

$\mathrm{LXA}_{4}$ was measured in nasal lavage supernatants by a commercially available ELISA kit (Neogen Crop, Lexington, Ky) according to the manufacturer's instructions. The lower detection limit of the assay was 0.02 $\mathrm{ng} / \mathrm{ml}$.

NK cells and eosinophil incubations 
For in vitro assays, peripheral blood was obtained from healthy subjects according to a protocol approved by the local Research Ethics Committee. Written informed consent was obtained from all subjects before their participation. Peripheral blood mononuclear cells (PBMCs) and granulocytes were isolated by density gradient centrifugation (Histopaque-1077 and Histopaque-1119 respectively; Sigma-Aldrich) as described earlier (12). NK cells, monocytes and T lymphocytes were isolated from PBMCs with a magnetically cell isolation kit (EasySep, Stemcell Technologies) according to the manufacturer's instructions. Eosinophils and neutrophils were further sorted from a granulocyte suspension with an ARIA flow cytometer (Becton Dickinson) as described earlier (18). In some selected experiments, CD $56^{\mathrm{dim}}$ and CD56 ${ }^{\text {bright }}$ NK cells were sorted with an ARIA flow cytometer (Becton Dickinson) from a total NK cell suspension obtained earlier with a magnetically NK cell isolation kit (EasySep, Stemcell Technologies). For NK cell/eosinophil cococultures, autologous human granulocytes or eosinophils were resuspended in RPMI 1640 medium (Gibco, BRL) supplemented with $10 \%$ heat-inactivated FCS (Gibco, BRL) and $2 \mathrm{mM} \mathrm{L-glutamine} \mathrm{(Gibco,} \mathrm{BRL),}$ in the absence or presence of total NK cells or CD56 ${ }^{\mathrm{dim}}$ and/or CD56 $6^{\text {bright }}$ NK cells (1:5 ratio of granulocytes/NK cells or CD $56^{\mathrm{dim}}$ or CD $56^{\text {bright }}$ subset) or autologous supernatants from earlier co-incubation assays ( 4 hours, $37^{\circ} \mathrm{C}, 5 \% \mathrm{CO}_{2}$ ). Apoptosis was assayed by FACS with annexin $\mathrm{V}$ and 7 -AAD staining (BD Pharmingen). For assays assessing superoxide generation, detection of superoxide radical anion was assessed by electron paramagnetic resonance (eScan, Bruker) in medium containing $200 \mu \mathrm{M}$ of CMH (1-hydroxyl-3methoxycarbonyl- 2,2,5,5-tetramethylpyrrolidine). In some experiments, cells were stimulated (10 min, 50 $\mathrm{ng} / \mathrm{ml}$ ) with Phorbol 12-myristate 13-acetate (PMA) (Sigma) and/or selectively exposed $\left(15 \mathrm{~min}, 37^{\circ} \mathrm{C}\right)$ to $\mathrm{LXA}_{4}(1,10$ or $100 \mathrm{nM})$ or vehicle (0.1\% ethanol) (Cayman Chemical).

\section{Statistical analysis}

Statistical analysis was performed using GraphPad Prism 8 Software (GraphPad Software, La Jolla, CA). Data are expressed as means \pm SEM. Comparison between allergen vs diluent at various time points were made by using 2-way ANOVA with Sidaks's multiple comparison test. Correlations were determined by using Pearson's correlation coefficient.In vitro data were analyzed by one-way-ANOVA with Tukey's multiple comparison test, two-tailed Student's $t$ test or multiplet -tests with Bonferroni correction. $\mathrm{P}$ values of less than .05 were considered statistically significant.

\section{Results}

\section{Nasal provocation with a single dose of allergen induces a typical nasal allergic inflammatory response that resolves spontaneously}

The nasal challenge with a single dose of grass pollen allergen induced typical allergic symptoms in all patients that resolved spontaneously. Clinical scores were highest 15 minutes after the administration of the allergen, falling progressively to pre-challenge levels by $48 \mathrm{hrs}$ after the challenge (Fig. 1, A ). A modest nasal obstruction was the longest lasting symptom (Fig. S1). No clinical response was observed after the diluent challenge. Total nasal lavage inflammatory cells and nasal lavage protein levels peaked at $1 \mathrm{hr}$ after the allergen challenge and returned progressively to pre-challenge values within $72 \mathrm{hrs}$ (Fig. 1, $B$ and $C$ ). No significant kinetics were observed after diluent challenges.

\section{NK cells appear early during the allergic inflammatory response}

Leukocyte sub-populations were furthermore identified in nasal lavage samples by FACS (the gating strategy is outlined in Fig. 2, A ). As expected, eosinophils were rapidly recruited, as early as $1 \mathrm{hr}$ post-challenge, and remained elevated for $48 \mathrm{hrs}$ after the challenge (Fig. 2, $B$ ). Neutrophils were the most abundant population, peaking $1 \mathrm{hr}$ after the challenge and returning to baseline levels at $24 \mathrm{hrs}$ (Fig. 2, $B$ ). Monocyte and lymphoid cell recruitment was also observed after the allergen challenge. Interestingly, NK cells were recruited as early as $1 \mathrm{~h}$ after the challenge, persisted for $6 \mathrm{hrs}$ before falling to their baseline levels. No significant leukocyte kinetics were observed after diluent challenges.

\section{NK cells interact with eosinophils}

We next observed that there was a positive relationship between the total number of nasal lavage NK cells 
and that of eosinophils 1, 6 and 24 hrs after allergen challenge (Figure 3, $A$ - $C$ ). No significant correlation was observed between NK cells and other leukocyte populations (data not shown). In vitro assays previously showed that peripheral blood NK cells are able to trigger apoptosis of autologous blood eosinophils (12). To determine whether soluble mediators were involved in NK cell-induced eosinophil apoptosis, we incubated freshly isolated eosinophils from healthy donors $\left(4 \mathrm{hrs}, 37^{\circ} \mathrm{C}\right)$ with supernatants from past autologous NK cell-eosinophil co-cultures kept at $-80^{\circ} \mathrm{C}$ (Fig $4, A$ ). These supernatants did not increase basal levels of eosinophils apoptosis, suggesting the need for a direct contact between eosinophil and NK cells. Based on their CD56 expression, two primary subsets of NK cells are found in human subjects, namely CD $56^{\mathrm{dim}}$ and CD56 $6^{\text {bright }}(10)$. The CD $56^{\text {bright }}$ NK cells are commonly described as cytokine-producing cells with poor cytotoxic ability, whereas CD $56^{\mathrm{dim}}$ NK cells are best known for their potent cytotoxic activity. To assess the role of one particular subtype is involved in eosinophil apoptosis, NK cells from healthy donors were sorted by FACS, based on their CD56 expression and incubated with autologous eosinophils $\left(4 \mathrm{hrs}, 37^{\circ} \mathrm{C}\right)($ Fig $4, B$, left panel ). Intriguingly, eosinophil apoptosis was not modified when eosinophils were incubated alone with either the CD56 $6^{\text {dim }}$ or CD $56^{\text {bright }}$ NK cell subset, suggesting a combined interaction of both subpopulations while inducing eosinophil apoptosis (Fig 4, B, right panel ).

Upon activation, eosinophils release many mediators, including highly reactive oxygen species that can damage surrounding tissues. Besides the potential pro-resolving effect of NK cells on eosinophils, activator effects by NK cells on eosinophils have also been reported (13). We first observed that isolated eosinophils were by far the most important producers of superoxide anion among different leukocyte populations involved in type 2 allergic inflammation, at rest and when activated (Fig 4, $C$ ). In contrast, NK cells were unable to release superoxide anion (Fig $4, C$ ). We next wanted to assess superoxide release by eosinophils at different time points after co-culture with NK cells. In the presence of NK cells, superoxide release from eosinophils was significantly reduced after $1 \mathrm{hr}$ of co-incubation. This inhibitor effect was overwhelmed after $4 \mathrm{hrs}$ of co-incubation (Fig 4,D), confirming the partial activator effect of NK cells on eosinophils (13).

\section{$\mathrm{LXA}_{4}$ is produced in the nasal mucosa after nasal allergen challenge}

Quantification of $\mathrm{LXA}_{4}$ levels in nasal lavage samples showed that $\mathrm{LXA}_{4}$ was produced in the nasal mucosa at baseline and significantly increased in the nasal lavage fluid $1 \mathrm{hr}$ after allergen challenge (Fig 5, A ). Increased $\mathrm{LXA}_{4}$ levels $1 \mathrm{hr}$ after allergen challenge correlated with the peak of nasal neutrophil infiltration (Fig 5, B ). Interestingly, LXA 4 levels also significantly increased at 48 and 72 hrs post-allergen challenge when compared to baseline levels and correlated at these time points to monocyte recruitment (Fig $5, C$ and $D)$.

\section{NK cells and LXA $_{4}$ inhibit superoxide production by eosinophils}

As anti-inflammatory effects of $\mathrm{LXA}_{4}$ on eosinophils and NK cells were previously reported (19), we next first determined whether $\mathrm{LXA}_{4}$ was able to modulate eosinophil superoxide release. Activated eosinophils significantly reduced their superoxide release in a dose-dependent manner when exposed to $\mathrm{LXA}_{4}$ (Fig $6, A$ ). When eosinophils were co-incubated with NK cells in the presence of $\mathrm{LXA}_{4}$, superoxide release was still inhibited in a dose-dependent manner $4 \mathrm{hrs}$ later (Fig 6, B ). This contrasts with the previously observed partial activator effect of NK cells on eosinophils (Fig $4, D$ ).

\section{Discussion}

In this study, we used a standardized nasal allergen provocation test with a single threshold dose that led to an acute self-limited allergic respiratory inflammatory response. Here we showed for the first time that NK cells are recruited to the nasal mucosa shortly after the initiation of the allergic inflammatory response and correlate with eosinophilic infiltration.

Natural killer (NK) cells are key members of the innate lymphocyte family with essential roles in host defense against pathogens as well as in malignancies (20). However, a role for NK cells in the regulation of type 2 immune responses and allergic diseases has been recently suggested. Indeed, experimental studies using murine models have shown that NK cells can participate in the regulation of allergen-induced eosinophilic 
airway inflammation (11, 21-23). Moreover, the presence of activated NK cells has been noted in patients with type 2 diseases, including asthma $(12,24)$, allergic chronic rhinosinusitis $(25,26)$ and atopic dermatitis (27). Whether NK cells enhance or limit type 2 immune responses is still debating.

In human in vitro experiments, it has been shown that human NK cells can promote apoptosis of autologous eosinophils but the molecular mechanisms underlying these events were only partially addressed $(13,28)$. In the present study, we confirmed that peripheral blood NK cells from healthy donors are able to induce the apoptosis of autologous peripheral blood eosinophils. As eosinophils have the capacity to release a broad range of toxic mediators into surrounding host tissues with consequent tissue damage, eosinophil apoptotic cell death followed by their subsequent phagocytosis by surrounding phagocytes is therefore critical for the successful resolution of allergic and asthmatic inflammation (29). Moreover, as suggested earlier (13, 28), we confirmed that NK cell-induced eosinophil apoptosis needed a direct contact between eosinophils and NK cells.

Besides potential inflammation pro-resolving effects, activator effects of NK cells on eosinophils have also been reported. In a previous study, human NK cells were shown to induce a dose-dependent in vitroactivation of autologous eosinophils after 3 and 12 hours of co-culture (13). Upon activation, eosinophils are able to release many mediators, including highly reactive oxygen species that can damage surrounding tissues and worsen inflammation (30). In the current study, we observed that among different leukocyte populations involved in type 2 allergic inflammation, healthy donor eosinophils from were by far the most important producers of superoxide anion. After $1 \mathrm{hr}$ of co-incubation with NK cells, the release of superoxide by eosinophils was significantly reduced. This inhibitor effect was overwhelmed after $4 \mathrm{hrs}$ of co-incubation, confirming a partial activator effect of NK cells on eosinophils.

Several classes of natural pro-resolving and anti-inflammatory chemical mediators are produced at sites of inflammation, including the respiratory mucosa (4). $\mathrm{LXA}_{4}$ is a lead member of a newly described class of pro-resolving lipid mediators derived from arachidonic acid that signals through ALX/FPR2 receptors (5). $\mathrm{LXA}_{4}$ has been reported to decrease allergic and asthmatic responses in mouse models (6). In the current study, we showed that $\mathrm{LXA}_{4}$ was already present in the nasal mucosa at baseline and significantly increased in the nasal lavage fluid shortly after the nasal allergen challenge.

$\mathrm{LXA}_{4}$ is produced by multistep enzymatic process resulting from lipoxygenase (LO) activities in different cell types. One of the main pathways of $\mathrm{LXA}_{4}$ synthesis involves lipoxygenation of arachidonic acid by 15LO in epithelial cells to generate 15(S)-hydroxyeicosatetranoic acid (15S-HETE) that can then be utilized as a substrate by the 5-LO expressed in neutrophils to synthesize $\mathrm{LXA}_{4}$ (31). In our study, neutrophils were the most abundant population recruited during the nasal allergic response, peaking $1 \mathrm{hr}$ after the allergen challenge and returning to baseline levels at $24 \mathrm{hrs}$. A such transient increase of neutrophils has been documented earlier after allergen challenge in animal models and human asthma but their role in allergic disease has been questioned (32-34). Interestingly, we observed an association between the $\mathrm{LXA}_{4}$ levels and the peak of nasal neutrophil infiltration, suggesting the involvement of neutrophils in $\mathrm{LXA}_{4}$ biosynthesis during the early respiratory allergic inflammatory response. After falling back to baseline levels, $\mathrm{LXA}_{4}$ significantly re-increased at 48 and $72 \mathrm{hrs}$ post-allergen challenge at time points where monocytes were present in the nasal lavage samples. An increasing number of studies have linked efferocytosis by macrophages and monocytes with their further production of pro-resolving mediators, including $\mathrm{LXA}_{4}(35)$.

Both NK cells (12) and eosinophils (36) express the LXA 4 receptor ALX/FPR2. Since anti-inflammatory effects of $\mathrm{LXA}_{4}$ on eosinophils were previously reported (19), we next determined whether $\mathrm{LXA}_{4}$ was able to modulate eosinophil superoxide release. Activated eosinophils significantly reduced their superoxide release in a dose-dependent manner when exposed to $\mathrm{LXA}_{4}$. When eosinophils were co-incubated with NK cells in the presence of $\mathrm{LXA}_{4}$, superoxide release was still inhibited in a dose-dependent manner $4 \mathrm{hrs} \mathrm{later,} \mathrm{in}$ contrast with the previously observed partial activator effect of NK cells on eosinophils 4 hrs later.

Our study underlines the complex network between cellular and molecular actors during resolution of allergic airway inflammation. Our data suggested that NK cells could represent an endogenous counterregulatory 
response to type 2 inflammation, as NK cells are recruited to the nasal mucosa during an allergic inflammatory response in parallel to eosinophil accumulation an are able to promote eosinophil apoptosis. The accumulation of neutrophils along with monocytes during the allergic inflammatory response may furthermore be an important regulatory feedback to initiate and promote resolution of allergic inflammation, as our data suggest involvement of these cells in $\mathrm{LXA}_{4}$ biosynthesis, a specific pro-resolving lipid mediator. Finally, $\mathrm{LXA}_{4}$ appeared to be essential in limiting superoxide release by eosinophils in the presence of NK cells.

Current treatment strategies in allergic airway diseases have focused on combating pathologic type 2 inflammation using either broad or selective immunosuppression. In the next future, the therapeutic paradigm could shift to the use of pharmacologic agents that enhance endogenous pro-resolving mechanisms. This presupposes a better understanding of the roles and interactions between cellular and molecular processes that drive the resolution of allergic inflammation.

In conclusion, here we report for the first time that NK cells are recruited to the nasal mucosa of subjects with allergy in response to nasal allergen challenge and we identify a synergistic role for NK cells and LXA4 in mediating resolution of allergic eosinophilic inflammation.

\section{Acknowledgments}

We thank Pr Seiamak Bahram and Dr Beatrice Uring-Lambert for access to flow cytometer.

\section{Funding Statement}

This study was supported by « Appel à Projets Jeunes Chercheurs 2013 GIRCI Est » and « Subvention 2015 de la Société Française d'Allergologie ».

\section{Conflict of interest}

The authors declare that there is no conflict of interest.

Author contributions : A.P. collected samples, designed and performed the experiments, collected and analyzed the data, and wrote the manuscript; G.W. designed and performed the experiments, C. L. performed the experiments, P.S., N.F., B.G. and F.D provided conceptual advice and edited the manuscript; C.B. designed the study, collected samples, performed the experiments, analyzed the data, and wrote the manuscript.

\section{References}

1. Bousquet J, Anto JM, Bachert C, Baiardini I, Bosnic-Anticevich S, Walter Canonica G, et al. Allergic rhinitis. Nat Rev Dis Primers. 2020;6(1):95.

2. Galli SJ, Tsai M, Piliponsky AM. The development of allergic inflammation. Nature. 2008;454(7203):44554.

3. Serhan CN. Treating inflammation and infection in the 21st century: new hints from decoding resolution mediators and mechanisms. FASEB J. 2017;31(4):1273-88.

4. Barnig C, Frossard N, Levy BD. Towards targeting resolution pathways of airway inflammation in asthma. Pharmacol Ther. 2018;186:98-113.

5. Serhan CN. Resolution phase of inflammation: novel endogenous anti-inflammatory and proresolving lipid mediators and pathways. Annu Rev Immunol. 2007;25:101-37.

6. Levy BD, De Sanctis GT, Devchand PR, Kim E, Ackerman K, Schmidt BA, et al. Multi-pronged inhibition of airway hyper-responsiveness and inflammation by lipoxin A(4). Nat Med. 2002;8(9):1018-23.

7. Karra L, Haworth O, Priluck R, Levy BD, Levi-Schaffer F. Lipoxin B(4) promotes the resolution of allergic inflammation in the upper and lower airways of mice. Mucosal Immunol. 2015;8(4):852-62. 
8. Vachier I, Bonnans C, Chavis C, Farce M, Godard P, Bousquet J, et al. Severe asthma is associated with a loss of LX4, an endogenous anti-inflammatory compound. J Allergy Clin Immunol. 2005;115(1):55-60.

9. Levy BD, Bonnans C, Silverman ES, Palmer LJ, Marigowda G, Israel E, et al. Diminished lipoxin biosynthesis in severe asthma. Am J Respir Crit Care Med. 2005;172(7):824-30.

10. Campbell KS, Hasegawa J. Natural killer cell biology: an update and future directions. J Allergy Clin Immunol. 2013;132(3):536-44.

11. Haworth O, Cernadas M, Levy BD. NK cells are effectors for resolvin E1 in the timely resolution of allergic airway inflammation. J Immunol. 2011;186(11):6129-35.

12. Barnig C, Cernadas M, Dutile S, Liu X, Perrella MA, Kazani S, et al. Lipoxin A4 regulates natural killer cell and type 2 innate lymphoid cell activation in asthma. Sci Transl Med. 2013;5(174):174ra26.

13. Awad A, Yassine H, Barrier M, Vorng H, Marquillies P, Tsicopoulos A, et al. Natural killer cells induce eosinophil activation and apoptosis. PLoS One. 2014;9(4):e94492.

14. Auge J, Vent J, Agache I, Airaksinen L, Campo Mozo P, Chaker A, et al. EAACI Position paper on the standardization of nasal allergen challenges. Allergy. 2018;73(8):1597-608.

15. Lebel B, Bousquet J, Morel A, Chanal I, Godard P, Michel FB. Correlation between symptoms and the threshold for release of mediators in nasal secretions during nasal challenge with grass-pollen grains. J Allergy Clin Immunol. 1988;82(5 Pt 1):869-77.

16. Naclerio RM, Proud D, Togias AG, Adkinson NF, Jr., Meyers DA, Kagey-Sobotka A, et al. Inflammatory mediators in late antigen-induced rhinitis. N Engl J Med. 1985;313(2):65-70.

17. Brooks CR, van Dalen CJ, Hermans IF, Douwes J. Identifying leukocyte populations in fresh and cryopreserved sputum using flow cytometry. Cytometry B Clin Cytom. 2013;84(2):104-13.

18. Wacht G, Poirot A, Charles AL, Radosavljevic M, Uring-Lambert B, de Blay F, et al. FACS - based isolation of human eosinophils allows purification of high quality RNA. J Immunol Methods. 2018;463:47-53.

19. Soyombo O, Spur BW, Lee TH. Effects of lipoxin A4 on chemotaxis and degranulation of human eosinophils stimulated by platelet-activating factor and N-formyl-L-methionyl-L-leucyl-L-phenylalanine. Allergy. $1994 ; 49(4): 230-4$.

20. Bjorkstrom NK, Ljunggren HG, Michaelsson J. Emerging insights into natural killer cells in human peripheral tissues. Nat Rev Immunol. 2016;16(5):310-20.

21. Korsgren M, Persson CG, Sundler F, Bjerke T, Hansson T, Chambers BJ, et al. Natural killer cells determine development of allergen-induced eosinophilic airway inflammation in mice. J Exp Med. 1999;189(3):55362 .

22. Ple C, Barrier M, Amniai L, Marquillies P, Bertout J, Tsicopoulos A, et al. Natural killer cells accumulate in lung-draining lymph nodes and regulate airway eosinophilia in a murine model of asthma. Scand J Immunol. 2010;72(2):118-27.

23. Mathias CB, Guernsey LA, Zammit D, Brammer C, Wu CA, Thrall RS, et al. Pro-inflammatory role of natural killer cells in the development of allergic airway disease. Clin Exp Allergy. 2014;44(4):589-601.

24. Timonen T, Stenius-Aarniala B. Natural killer cell activity in asthma. Clin Exp Immunol. 1985;59(1):8590 .

25. Mesdaghi M, Vodjgani M, Salehi E, Hadjati J, Sarrafnejad A, Bidad K, et al. Natural killer cells in allergic rhinitis patients and nonatopic controls. Int Arch Allergy Immunol. 2010;153(3):234-8.

26. El-Shazly AE, Doloriert HC, Bisig B, Lefebvre PP, Delvenne P, Jacobs N. Novel cooperation between CX3CL1 and CCL26 inducing NK cell chemotaxis via CX3CR1: a possible mechanism for NK cell infiltration 
of the allergic nasal tissue. Clin Exp Allergy. 2013;43(3):322-31.

27. Mack MR, Brestoff JR, Berrien-Elliott MM, Trier AM, Yang TB, McCullen M, et al. Blood natural killer cell deficiency reveals an immunotherapy strategy for atopic dermatitis. Sci Transl Med. 2020;12(532).

28. Pesce S, Thoren FB, Cantoni C, Prato C, Moretta L, Moretta A, et al. The Innate Immune Cross Talk between NK Cells and Eosinophils Is Regulated by the Interaction of Natural Cytotoxicity Receptors with Eosinophil Surface Ligands. Front Immunol. 2017;8:510.

29. Felton JM, Lucas CD, Dorward DA, Duffin R, Kipari T, Vermeren S, et al. Mer-mediated eosinophil efferocytosis regulates resolution of allergic airway inflammation. J Allergy Clin Immunol. 2018;142(6):188493 e6.

30. Klion AD, Ackerman SJ, Bochner BS. Contributions of Eosinophils to Human Health and Disease. Annu Rev Pathol. 2020;15:179-209.

31. Chavis C, Vachier I, Chanez P, Bousquet J, Godard P. 5(S),15(S)-dihydroxyeicosatetraenoic acid and lipoxin generation in human polymorphonuclear cells: dual specificity of 5-lipoxygenase towards endogenous and exogenous precursors. J Exp Med. 1996;183(4):1633-43.

32. Ohkawara Y, Lei XF, Stampfli MR, Marshall JS, Xing Z, Jordana M. Cytokine and eosinophil responses in the lung, peripheral blood, and bone marrow compartments in a murine model of allergen-induced airways inflammation. Am J Respir Cell Mol Biol. 1997;16(5):510-20.

33. Tomkinson A, Cieslewicz G, Duez C, Larson KA, Lee JJ, Gelfand EW. Temporal association between airway hyperresponsiveness and airway eosinophilia in ovalbumin-sensitized mice. Am J Respir Crit Care Med. 2001;163(3 Pt 1):721-30.

34. Lommatzsch M, Julius P, Kuepper M, Garn H, Bratke K, Irmscher S, et al. The course of allergen-induced leukocyte infiltration in human and experimental asthma. J Allergy Clin Immunol. 2006;118(1):91-7.

35. Sugimoto MA, Vago JP, Perretti M, Teixeira MM. Mediators of the Resolution of the Inflammatory Response. Trends Immunol. 2019;40(3):212-27.

36. Pazdrak K, Straub C, Maroto R, Stafford S, White WI, Calhoun WJ, et al. Cytokine-Induced Glucocorticoid Resistance from Eosinophil Activation: Protein Phosphatase 5 Modulation of Glucocorticoid Receptor Phosphorylation and Signaling. J Immunol. 2016;197(10):3782-91.

Figure 1. Nasal provocation with a single dose of allergen induces a rapid nasal allergic inflammatory response that resolves spontaneously over $72 \mathrm{hrs}$. Subjects $(\mathrm{n}=20)$ suffering from confirmed seasonal grass pollen rhinitis were challenged intranasally with a single threshold dose of a grass pollen extract or diluent. A,Nasal symptoms were assessed at baseline and at different time points (15 min, $30 \mathrm{~min}, 1,6,24,48$ and $72 \mathrm{hrs}$ ) after nasal challenge. The total nasal symptom score (TNSS) represents the cumulative score of sneezing, nasal running (rhinorrhea), itching and nasal blockage (obstruction) each scored from 0 to 3 using a questionnaire. $\mathbf{B}$, Total inflammatory cells and $\mathbf{C}$, protein levels were measured in nasal lavage samples collected at the indicated time points after allergen challenge or diluent. Data are displayed as mean \pm SEM; ${ }^{*} P<0.05,{ }^{* *} P<0.01,{ }^{* * * *} P<0.0001 ; 2$-way ANOVA with Sidaks's multiple comparison test was used to compare allergen $v s$ diluent at various time points.

Figure 2. NK cells appear early during the allergic inflammatory response. A, Representative dot plots for flow cytometric sequential gating of leukocyte populations in nasal lavage. First, doublets were excluded, then CD45- events and subcellular debris. Leukocyte populations (granulocytes, lymphoid cells and monocytes) were identified on the basis of CD14 expression and side scatter. Eosinophil and neutrophils were identified among granulocytes on the basis of absence or presence of CD16 expression, respectively. NK cells and $\mathrm{T}$ cells were identified among the lymphoid cell population as CD3 ${ }^{-} \mathrm{NKp} 46^{+}$cells and $\mathrm{CD} 3^{+} \mathrm{NKp}^{-} 6^{-}$ cells, respectively.B , Time course of neutrophils, eosinophils, NK cells, lymphoid cells, T lymphocytes and monocytes following allergen nasal challenge or diluent. Data are displayed as mean $+-\mathrm{SEM} ;{ }^{*} P<0.05$; 
${ }^{* *} P<0.01 ;{ }^{* * *} P<0.001 ; 2$-way ANOVA with Sidaks's multiple comparison test was used to compare allergenvs diluent at various time points.

Figure 3. NK cells correlate with eosinophils during the allergic inflammatory response. A, Correlation between total NK cells and eosinophils 1,6 and $24 \mathrm{~h}$ after the allergen challenge (Pearson correlation $r$ value and significance are noted).

Figure 4. NK cells are able to induce apoptosis in autologous eosinophils and to inhibit superoxide production by eosinophils.A , Peripheral blood eosinophils were incubated ( $4 \mathrm{hrs}, 37 \mathrm{degC}$ ) with medium alone, autologous supernatants from past eosinophil cultures, autologous supernatants from past NK celleosinophil co-cocultures (1:5 ratio) and autologous peripheral blood NK cells (1:5 ratio). Eosinophil apoptosis was assayed by FACS with annexin-V and 7-AAD staining. Results are expressed as means +- SEM, $n=$ 6 individual healthy donors; ${ }^{* * * *} \mathrm{P}<0.0001$; one-way ANOVA with Tukey's multiple comparison test $\mathbf{B}$, CD56 ${ }^{\mathrm{dim}}$ and CD56 ${ }^{\text {bright }}$ NK cells were sorted by flow cytometry (left panel) and co-incubated (4 hrs, $\left.37 \mathrm{degC}\right)$ alone or together with autologous eosinophils (1:5 ratio) (right panel). Eosinophil apoptosis was assayed by FACS with annexin-V and 7-AAD staining. Results are expressed as means +- SEM, $n=5$ individual healthy donors, ${ }^{* * * *} \mathrm{P}<0.0001$, one-way ANOVA with Tukey's multiple comparison test.C , Superoxide release was assessed by electron paramagnetic resonance (EPR) in different leukocyte population involved in type 2-mediated allergic inflammation at rest (vehicle) and when activated by phorbol myristate acetate (PMA) (10 min, $50 \mathrm{ng} / \mathrm{mL}$ ). Results are expressed as means $+-\mathrm{SEM}, n=5$ individual healthy donors, ${ }^{*} \mathrm{P}<0.05$, at rest (vehicle) when cells are compared to each other; ${ }^{* *} \mathrm{P}<0.05$, after PMA when cells are compared to each other; ${ }^{\mathrm{PS}} \mathrm{P}<0.05$, when cells are compared between rest (vehicle) and PMA; multiple $t$ -tests with Bonferroni correction. D , NK cells induce a significant reduction of superoxide production by FACS isolated eosinophils $1 \mathrm{hr}$ after co-incubation $(37 \mathrm{degC}, 1: 5$ ratio) that is overwhelmed after $4 \mathrm{hrs}$ of co-incubation. Results are expressed as means +- SEM, $n=7$ individual healthy donors, ${ }^{*} \mathrm{p}<0.05$, paired Student's $t$ test.

Figure $5 . \mathrm{LXA}_{4}$ is produced in the nasal mucosa after nasal allergen challenge and correlate to neutrophil and monocyte recruitment. A , $\mathrm{LXA}_{4}$ concentrations were quantified by ELISA in nasal lavage samples. Values are expressed as means + - SEM; ${ }^{*} P<0.05$ compared to diluent, ${ }^{\$} \mathrm{P}<0.05$ compared to baseline levels; 2-way ANOVA with Sidaks's multiple comparison test was used to compare allergen vs diluent at various time points and 2-way ANOVA with Tukey's multiple comparison test was used to compare levels vs baseline levels. B, Correlation of $\mathrm{LXA}_{4}$ levels and neutrophil counts $1 \mathrm{hr}$ after allergen challenge (Pearson correlation $r$ value and significance are noted). C , Correlation of $\mathrm{LXA}_{4}$ levels and monocyte levels $48 \mathrm{hrs}$ (left panel) and $72 \mathrm{hrs}$ (right panel) after allergen challenge (Pearson correlation $r$ value and significance are noted).

Figure $6 . \mathrm{LXA}_{4}$ is essential to inhibit NK cells triggered eosinophil superoxide release in vitro . A , Eosinophils were pre-treated $(15 \mathrm{~min})$ with increasing doses of $\mathrm{LXA}_{4}(1,10$ or $100 \mathrm{nM}$ or vehicle [0.1\% ethanol]) and then stimulated with PMA (10 min, $50 \mathrm{ng} / \mathrm{mL})$, superoxide release was assessed by EPR. Results are expressed as means $+-\mathrm{SEM} ; n=6$ individual healthy donors; ${ }^{*} \mathrm{p}<0.05$ compared to vehicle, ${ }^{\mathrm{PS}} \mathrm{p}<0.05$ compared to $1 \mathrm{nM}$; one-way ANOVA with Tukey's multiple comparison test. B , Eosinophils were pre-tretated $(15 \mathrm{~min})$ with $\mathrm{LXA}_{4}(10,100 \mathrm{nM}$ or vehicle) and co-incubated with NK cells $(37 \mathrm{degC}$, 1:5 ratio). Eosinophils showed still a dose-dependent reduction of superoxide production after $4 \mathrm{hrs}$ of coincubation. Results are expressed as means +- SEM, $n=3$ individual healthy donors; ${ }^{*} \mathrm{P}<0.05$; multiple $t$-tests with Bonferroni correction.

Figure S1. Detailed clinical scores composing the total nasal symptom score (TNSS). A, Nasal sneezing, $\mathbf{B}$, nasal itching, $\mathbf{C}$, nasal running (rhinorrhea) and $\mathbf{D}$, nasal blockage (obstruction). Data are displayed as mean +- SEM; ${ }^{*} P<0.05,{ }^{* * *} P<0.001,{ }^{* * * *} P<0.0001 ; 2$-way ANOVA with Sidaks's multiple comparison test was used to compare allergen $v s$ diluent at various time points.

\section{Hosted file}

image1.emf available at https://authorea.com/users/414290/articles/522364-nk-cells- 
and-lipoxin-a4-promote-resolution-of-eosinophilic-inflammation-after-nasal-allergenchallenge

\section{Hosted file}

image2.emf available at https://authorea.com/users/414290/articles/522364-nk-cellsand-lipoxin-a4-promote-resolution-of-eosinophilic-inflammation-after-nasal-allergenchallenge

\section{Hosted file}

image3.emf available at https://authorea.com/users/414290/articles/522364-nk-cellsand-lipoxin-a4-promote-resolution-of-eosinophilic-inflammation-after-nasal-allergenchallenge

\section{Hosted file}

image4.emf available at https://authorea.com/users/414290/articles/522364-nk-cellsand-lipoxin-a4-promote-resolution-of-eosinophilic-inflammation-after-nasal-allergenchallenge

\section{Hosted file}

image5.emf available at https://authorea.com/users/414290/articles/522364-nk-cellsand-lipoxin-a4-promote-resolution-of-eosinophilic-inflammation-after-nasal-allergenchallenge

\section{Hosted file}

image6.emf available at https://authorea.com/users/414290/articles/522364-nk-cellsand-lipoxin-a4-promote-resolution-of-eosinophilic-inflammation-after-nasal-allergenchallenge

\section{Hosted file}

image7.emf available at https://authorea.com/users/414290/articles/522364-nk-cellsand-lipoxin-a4-promote-resolution-of-eosinophilic-inflammation-after-nasal-allergenchallenge 